Vol. 1, No. 1, 2020

https://doi.org/

P. Kosobutskyy

Lviv Polytechnic National University

\title{
METHODICAL ASPECTS OF STATISTICAL MODELING OF TWO-DIMENSIONAL SYSTEMS OF RANDOM VARIABLES
}

(c) Kosobutskyy P., 2020

According to the analysis of literature sources, the statistical processing of measurement results is not always given due attention. Unfortunately, appropriate algorithms are often limited to simplified statistical procedures, without the proper justification of the objective function, including to check the quality of processing of random data. Therefore, the author plans to publish a series of articles on statistical modeling, which will include the results of original research by the author and others. In this article are considered the methodological aspects of statistical modeling of two-dimensional systems with random data, physical substantiation of correlation regularities of statistical relations between random variables is given, since or the problem of establishing the law of distribution of random variable has practical interest from the point of view of modeling statistical regularities of model "signal + noise".

Keywords: Two-Dimensional Systems, Conditional probabilities. Dependent and independent, Jacobian determinants, Correlation, and Transformation of Tow-Dimensional

\section{Introduction}

In statistical research, the functions of not just one, but two or more random variables (RVs), are used. Thus, the analysis of variance mainly investigates mutually statistically independent RVs that are normally distributed, such as $X=X_{1}{ }^{2}+X_{2}{ }^{2}+. .+X_{n}{ }^{2}$ and $Y=Y_{1}{ }^{2}+Y_{2}{ }^{2}+. .+Y_{m}{ }^{2}$. In Snedecor's statistics, the probabilistic regularities of RVs $\frac{n}{m} \frac{X}{Y}$ are used, and in Fisher's statistics, the probabilistic regularities of type $Z=\log \frac{1}{2}\left(\frac{n}{m} \frac{X}{Y}\right)$ are used. The probability density function of the RVs as $\frac{X}{\sqrt{Y_{1}^{2}+Y_{2}^{2}+. .+Y_{m}^{2}}}$ expectation is equal to zero, and due to homogeneity, the variance is not playing any role [1].

It is believed that the work [2] gave rise to a theory of summation of independent RVs. It was found that some linear operations, such as summation and finding the minimum and maximum values of RVs, generate $\mathrm{RVs}$, the distribution of which converges to several limit distributions, such as Gaussian distribution $\frac{1}{\sqrt{\pi} \sigma_{X}}\left(x_{1}+x_{2}+\ldots+x_{k}-m_{X} \cdot k\right)$ (normal distribution), stable distributions $\frac{1}{k^{1 / \alpha}}\left(x_{1}+x_{2}+\ldots+x_{k}\right)$ (StableDistribution), and Fisher-Tippet-Gnedenko distribution $a_{k} \max \left(x_{1}, x_{k}, \ldots, x_{k}\right)+b_{k}$ (MinStableDistribution and MaxStableDistribution) [3]. In the above examples, random phenomena are described by several normally interrelated random numbers in the general case, where the probabilistic characteristics of the RV system should be researched. We will turn your attention only to the analysis of the system of two continuous RVs, although they can be discrete, continuous, and mixed, depending on the types of the RV included in these systems. 
In statistical modeling, the properties of the so-called self-reproduction of the distribution laws are widely used. Thus, the sum of normally distributed RVs is also normally distributed; according to Cauchy law, the sum of Cauchy-distributed RVs, etc. The property of self-reproduction of the distribution laws allows determining the type of the probability density function of an error by the given type of the error as a random argument function and the known distribution of the arguments [4]. This is based on the fact that if there are no correlations between components of the error composition, then the distribution of the sum of normally distributed errors is distributed as close as possible to normal. This pattern is true more accurately, the greater the number of RVs that add up. Therefore, regardless of the laws, the individual errors are subject to, the features of the distributions in the sum of a large number of summands are dropped out of the equation, and the sum turns out to be subject to normal distribution law. The joint distribution of the composition of two independent RVs has been studied by many authors [5].

\section{Methodical analysis and discussion}

Two-Dimensional Systems of $R V \boldsymbol{~ s}$. In many practical problems, we have to deal with not just one $\mathrm{RV}$, but with a system of RVs. Thus, a thermodynamic system in a state of thermal balance is described by at least three parameters - volume, pressure, and temperature. Electrical and thermal systems are described by such parameters as voltage, the resistance of an active component, and the current passing through it. In deformation systems, the physical processes of energy conversion are determined by the external force applied, reaction to this force, and the resilience of the component, etc. [6,7].

Physical variables in the system can be independent of each other or related to each other to a certain extent. For characteristics of the probabilistic and statistical regularities of a system of two or more RVs, the same joint distribution and joint moments, etc., are introduced as for one-dimensional RVs. The relationship between the joint two-dimensional probability density function $f_{(X, Y)}(x, y)$ and the joint moment of the $k, l$ order is put down as [7]

$$
\overline{x^{k} y^{l}}=\int_{-\infty}^{+\infty} x^{k} y^{l} \cdot f_{(X, Y)}(x, y) d x d y
$$

Both for one-dimensional and multi-dimensional RVs, the setting of all moments is similar to setting the probability density function.

In a two-dimensional system with joint probability density function $f_{(X, Y)}(x, y)$, the probability of distribution of each of the components of the RV expresses the so-called marginal (individual) probabilities with probability density function $f_{X}(x), f_{Y}(y) \square$ :

$$
f_{X}(x)=\int_{-\infty}^{+\infty} f_{(X, Y)}(x, y) d y, \quad f_{Y}(y)=\int_{-\infty}^{+\infty} f_{(X, Y)}(x, y) d x
$$

Thus, we should speak about both the individual independence of RVs and joint independence for an arbitrary set of RVs. Thus, a two-dimensional set of RVs is independent, if the joint probability density function is equal to the product of marginal probability density functions of RVs [8]

$$
F_{(X, Y)}(x, y)=F_{X}(x) F_{Y}(y) \text { or } \mathrm{P}(X\langle x, Y<y)=\mathrm{P}(X\langle x) \mathrm{P}(Y<y)
$$

then such explosives RVs $X$ and $Y$ are independent, and the two-dimensional the distribution function $F_{X Y}(x, y)$ is factorized. For independent RVs, the common density function $f_{(X, Y)}(x, y)$ is expressed by the product of one-dimensional densities of marginal probability distributions $f_{X}(x)$ and $f_{Y}(y)$ from RVs $X$ and $Y$ :

$$
f_{(X, Y)}(x, y)=f_{X}(x) \cdot f_{Y}(y) .
$$

In general, it is impossible to solve the inverse problem, that is, to restore the joint distribution of the components. 
Then in this case (4), we have:

$$
f_{Z}(z)=\int_{-\infty}^{+\infty} f_{X}(x) f_{Y}(z-x) d x \text { or } f_{Z}(z)=\int_{-\infty}^{+\infty} f_{X}(z-y) f_{Y}(y) d y .
$$

Only in this case, the so-called inverse problem can be solved; according to the known RV distribution laws, we can find a joint distribution law, as the distribution law itself does not indicate the nature of the relationship between the RVs. Let us recall that it can be both functional and statistical (probabilistic). Independent RVs are uncorrelated, whereas their uncorrelatedness does not stand for their independence. If the correlation moment is equal to zero this means only that there is no linear relationship, but does not exclude any other relationship. Dependent variables can be correlated and uncorrelated. The formulas (3) and (4) are called the product rule of distribution laws. To fully characterize the distribution of a two-dimensional system, knowing the distribution of each of the RVs in it is not enough; one should also know the dependence between the RVs, which is characterized by conditional probabilities ${ }^{\square)}$.

Conditional probabilities. Dependent and independent $R V s$. In the probability theory, concepts of dependence and independence of RVs of probabilistic (stochastic) nature are important, as RV $Y$ is related to $\mathrm{RV} X$ by conditional dependence. This means that by knowing the value $X$ you cannot specify the exact value $Y$, but only indicate its distribution law, which depends on the value $X$. RVs are independent within a system $(X, Y)$ of the events $X<x$ and $Y<y$ are not dependent on random $x, y$. Note that we are talking about the statistical independence of aggregate RVs, which in this case forms a two-dimensional system. Thus, even the pairwise independence of the RVs does not presuppose the independence of aggregate RVs. Thus, if RVs $X$ and $Y$ are independent, then both the events related to falling of the RVs at the intervals $(-\infty, x)$ and $(-\infty, y)$ random intervals $\left[x_{1}, x_{2}\right)$ and $\left[y_{1}, y_{2}\right)$ are independent.

Analysis of the references shows that the independence of the RVs is simply declared in the majority of cases. From the point of view of physics, the pressure is an average perpendicular component of the force of the system applied to the regional unit of its margin of separation with its environment (for example, a thermostat):

$$
P=\sum_{i} \frac{d\left(m_{i} \vartheta_{i}\right)}{d t}
$$

The temperature of the system introduced by Boltzmann as a measure of its heating in terms of the smallness of the mass $m_{i}$ of gas molecules, atoms, and microparticles with proportional equality of the type of temperature

$$
\sum_{i} \frac{1}{2} m_{i} \vartheta_{i}^{2}=k T
$$

Thus, based on the physical nature, pressure, and temperature, including their random values, are dependent. However, the pressure and temperature are mostly statistical characteristics, the values of which reflect the regularities of collective systems. But a researcher measures pressure with one tool, and temperature with another tool. Therefore, if he carries out this procedure independently of the other one, and if the measurement conditions remain unchanged, then, taking into account the fluctuation processes, random values $P, T$ can be considered independent. The same model can be adopted for the analysis of the measured values of current and voltage $U, I$ on the electrical circuit component with resistance $R$. However, we emphasize that at the same time both parameters are caused by the same physical process at a given moment in time - a change in the number of electrons transferred by the electric field, which

Zener diode voltage regulator [9] can be a physical model of conditional probability. 
changes due to the processes of irreversible dissipation of their energy on defects. But, for statistical analysis, in particular, for example, for the establishment of the distribution law of random values $R$ concerning $I$, in most cases, it is assumed that $U, I$ are normally distributed, as will be discussed in more detail below.

According to [10], if the variables $\xi_{1}, \xi_{2}, \ldots, \xi_{n}$ are random values, then each aggregate value $\left(\xi_{1}, \xi_{2}, \ldots, \xi_{n}\right)$ is also a random value. The value $\left(\xi_{1}, \xi_{2}, \ldots, \xi_{n}\right)$ represents a joint distribution of value $\xi_{1}, \xi_{2}, \ldots, \xi_{n}$. At the same time, each aggregate RV, as well as a single RV, has a one-dimensional probability distribution. Joint distribution occurs in the case of repeated measurements. In the above example, the joint distribution occurs, if the RVs $U$ and $I$ form a random value as a product $P=U \cdot I$. Therefore,

Thus, if two RVs are dependent, then the information about the actual value of one of them will change our understanding of the distribution of another variable. Therefore, a concept of conditional distribution law was introduced for dependent RVs. The distribution calculated under the condition that another RV takes a certain value is called conditional. For continuous RV, the distribution law of RVs $X$ calculated under the condition that another $\operatorname{RV} Y$ takes a certain value $Y=y$ [2] is called conditional (integral $F(x \mid y)$ and differential $f(x \mid y))$ distribution law:

$$
f_{Y \mid X}(y \mid x)=\frac{1}{f_{X}(x)} \frac{\partial F_{X, Y}(x, y)}{\partial x}, \quad f_{X \mid Y}(x \mid y)=\frac{1}{f_{Y}(y)} \frac{\partial F_{X, Y}(x, y)}{\partial y} /
$$

Here the two-dimensional RVs $(X, Y)$ is continuous if there is a negative differential function of the common distribution

$$
f_{(X, Y)}(x, y)=\frac{d^{2}}{d x d y} F_{(X, Y)}(x, y)
$$

for which

$$
\left\{\begin{array}{l}
f_{X}(x)=\int_{-\infty}^{+\infty} f(x, y) d y \\
f_{Y}(y)=\int_{-\infty}^{+\infty} f(x, y) d x
\end{array}\right.
$$

Then the integral function of the common probability distribution will be equal to

$$
F_{(X, Y)}(x, y)=\mathrm{P}\left(-\infty\left\langleX \left\langle x<+\infty, Y\langle y)=\int_{-\infty}^{x} \int_{-\infty}^{y} f_{(X, Y)}(x, y) d x d y\right.\right.\right.
$$

Thus, we can determine the distribution law of the system by knowing the distribution law of one of the values included in the system, and the conditional distribution law of the other RV.

The two-dimensional differential probability element $d F_{(X, Y)}(x, y)$ is equal to

$$
f_{(X, Y)}(x, y) d x d y=\mathrm{P}((x<X<x+d x) \cdot(y<Y<y+d y)),
$$

that is, it is expressed through the product of the probabilities of the components. According to the multiplication theorem on probability [11]

$$
f_{(X, Y)}(x, y)=f_{X}(x) \cdot f_{Y \mid X}(y \mid x),
$$

the probability of the product of probabilities is equal to the following:

$$
f_{(X, Y)}(x, y) d x d y=f_{X}(x) d x \cdot f_{Y \mid X}(y \mid x) d y,
$$


Therefore, the probability density function of the system of two RVs is equal to the probability density function of one of the values included in the system multiplied by the conditional probability density function of another value calculated on the condition that the first quantity has a certain value. Integral of (14) is equal

$$
\int_{-\infty}^{+\infty} f_{(X, Y)}(x, y) d y=f_{X}(x)
$$

or a similar expression

$$
\int_{-\infty}^{+\infty} f_{(X, Y)}(x, y) d x=f_{Y}(y)
$$

allows calculating the distributions $f_{X}(x)$ and $f_{Y}(y)$, only if a two-dimensional distribution $f_{(X, Y)}(x, y)$ is set, and if we are not interested in the value of the second RV. For example, suppose if an ideal gas is located between two solid walls $x=a$ and $x=b(a\langle b)$, then the distribution of the coordinate $X$ of the ideal gas molecule is independent of $x$ :

$$
f(x)=\left\{\begin{array}{l}
\frac{1}{b-a} \quad \text { if } \quad a<x<b, \\
0 \quad \text { if } \quad a<x \text { or } x>b .
\end{array}\right.
$$

The RV $X$ is independent of the RV $Y$ if the law of distribution of the quantity $X$ does not depend on the value of the quantity $Y$. For continuous RVs, the condition of independence $Y$ can be put down as follows:

$$
f_{X \mid Y}(x \mid y)=f_{X}(x)
$$

at random $x$. Similarly, the condition of independence of $Y$ of $X$ has the following form:

$$
f_{Y \mid X}(y \mid x)=f_{Y}(y)
$$

at random $y$. Thus, if the conditional and unconditional distributions of RVs coincide, then these random variables are independent. Illustration of regularities of density function formation (19) from two directions of observation is shown in Fig. 1. The calculation parameter is chosen at random.

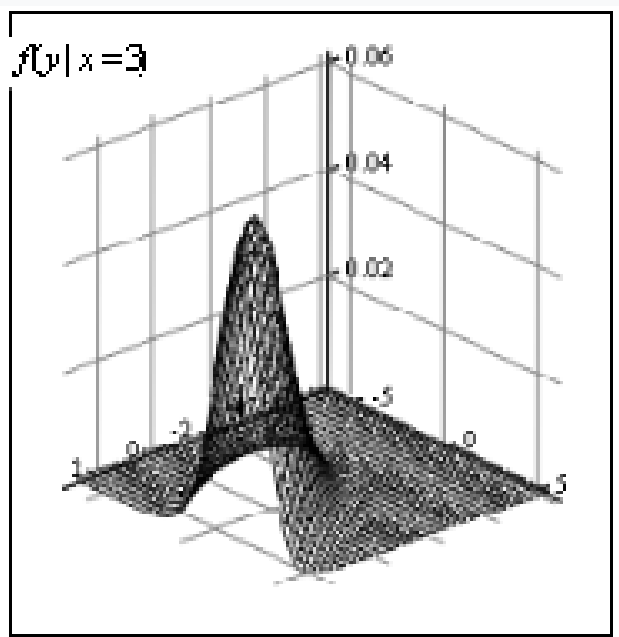

$\mathbf{f X Y}$

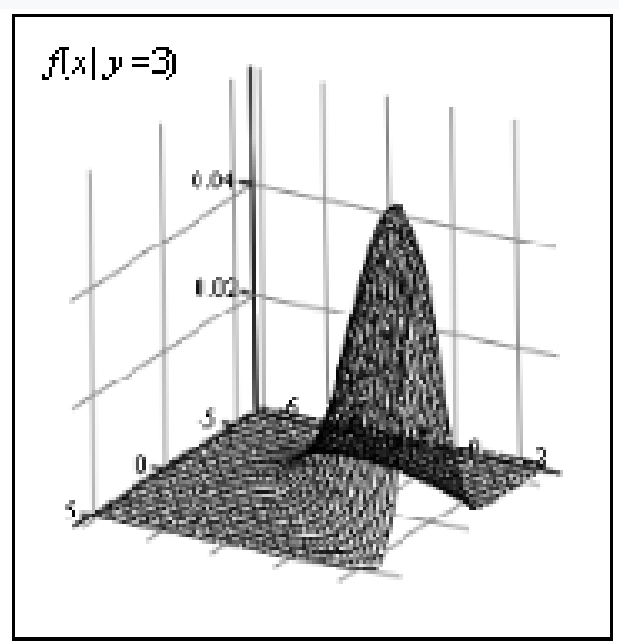

$\mathrm{EXI}$

Fig. 1. Illustration of regularities of density function formation (19) from two directions of observation 
The conditional distributions are characterized by the conditional mathematical expectation

$$
E_{X \mid Y=y}=\int_{-\infty}^{\infty} x f(x \mid y) d x
$$

and conditional standard deviation

$$
\sigma_{X \mid Y=y}{ }^{2}=\int_{-\infty}^{\infty}\left(x-m_{X \mid Y=y}\right)^{2} f(x \mid y) d x=\int_{-\infty}^{\infty} x^{2} f(x \mid y) d x=-m_{X \mid Y=y}{ }^{2} .
$$

Thus, if RV $X \sim N\left(m_{X}, \sigma_{X}\right)$, then the conditional mathematical expectation of normally distributed $\mathrm{RV}$ is equal to $m_{X}-\sqrt{\frac{2}{\pi}} \sigma_{X}$, and is less than the unconditional mathematical expectation [2]. Features of formation of probability density functions of dependent and independent RVs in a flat two-dimensional system are reflected in [12]. Fundamental studies of correlation and dependence of RVs given in [13].

\section{Correlation and Transformation of Tow-Dimensional RVs}

Correlation between the $R V s$ ). The mean and the variance are convenient features of RVs. The relationship between the two RVs $X$ and $Y$ is probabilistic, that is, when one of the changes, the other one will not change deterministically, but a trend to change will occur. According to (1), the following equation is justified for a two-dimensional system of independent RVs:

$$
\overline{x^{k} y^{l}}=\overline{x^{k}} \cdot \overline{y^{l}} \text {. }
$$

Therefore, to test the hypothesis of the independence of RVs, we should verify the validity of the equation (22) for arbitrary positive integers $k$ and $l$. Since this is very hard to do, in practice, the validity of the equation (22) is verified for any values $k$ and $l$. The difference between the left and right parts in (22) serves as a measure of a statistical relationship, which is called covariance in the case of two RVs $(k=1$ and $l=1)$ :

$$
\mathrm{K}_{X Y}=\overline{x y}-\bar{x} \cdot \bar{y}
$$

The formula (23) expresses the degree of probabilistic relationship between the two RVs in the language of mean RVs $X$ and $Y$. If $X=Y$, then the correlation is equal to the variance

$$
\mathrm{K}_{X Y}=\overline{X^{2}}-\bar{X}^{2} \text {. }
$$

Normalized covariance:

$$
\rho=\rho_{X Y}=\frac{\mathrm{K}_{X Y}}{\sqrt{\mathrm{K}_{X X} \cdot \mathrm{K}_{Y Y}}}=\frac{\overline{x y}-\bar{x} \cdot \bar{y}}{\sigma_{X} \sigma_{Y}}
$$

is called correlation coefficient between the variables $X$ and $Y$.

Unlike covariance, the normalized variable $\rho$ is dimensionless, that is why it is more convenient for practical use; its absolute values for independent RVs vary from zero to \pm 1 at $Y= \pm X$. Therefore, the correlation coefficient characterizes the degree of linear dependence of RVs. For a normally distributed two-dimensional system, the Pearson correlation coefficient is used for correlation studies [15].

If the distribution of $\mathrm{RVs}$ is symmetric with respect to the zero point (origin point), then the mathematical expectation of the type $X^{2 k+1} \mathrm{RVs}$ is equal to zero and the covariance is $\operatorname{cov}\left(X, X^{2}\right)=E_{X^{3}}-E_{X} E_{X^{2}}=0 \Rightarrow \operatorname{cov}\left(X, X^{2}\right)=0$, while the relationship between the RVs $X$ and $X^{2}$ is non-random. Therefore, the equality of the correlation function to zero is not a sufficient condition for the independence of RVs [14] 
The variance of correlated RVs is calculated by the formula

$$
D[X+Y]=D[X]+D[Y]+2 \rho \sigma_{X} \sigma_{Y},
$$

where the third summand expresses the mutual correlation moment depending on the parameters $\rho, \sigma_{X}$ and $\sigma_{Y}$. If RVs are independent, then they are uncorrelated $\rho=0$. The inverse proposition is not true. You can build RVs, which are not correlated, yet are dependent[16] thus, the equality to zero of the correlation coefficient $\rho=0$ still does not imply the independence of RVs [17], a visual illustration is shown in Figure 2. In this figure, its left side shows a chaotic scattering of points with statistically independent coordinates. We see that the trend of the regularity of distribution is absent. The right part shows a similar distribution when there is a correlation between the $\mathrm{X}$ and $\mathrm{Y}$ coordinates. Then it is possible to draw some averaged curve - the distribution trend.

An example of an uncorrelated dependence of RVs can be a nonlinear relationship $Y=a X^{2}$. The concept of correlation of RVs is close to the concept of dependence (in terms of statistics), but they are not equivalent. The only exception to this rule is the case of two normally distributed RVs when the concept of correlation and dependence are equivalent. Thus, if $\left|\mathrm{K}_{X Y}\right| \neq 0$, then the RVs are interdependent. But $\mathrm{K}_{X Y}$ displays the information about the scattering of a random point $(X, Y)$ relative to a fixed point $(M[X], M[Y])$. Therefore, if the variance of one of the RVs is small, then their covariance will be close to zero, no matter how strong is the relationship between them. Other regularities of uncorrelated dependent RVs are available in the works [16].

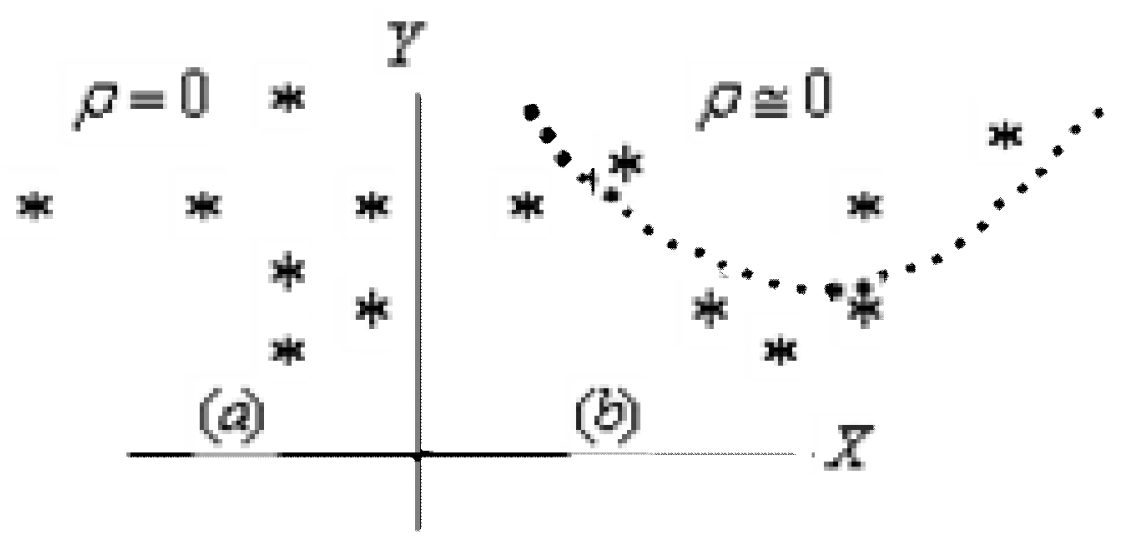

Fig. 2. The illustration of the chaotic scattering of points with statistically independent of coordinates $X$ and $Y$ (left part) and a correlation coordinates (right part)

Covariance characterizes not only the degree of dependence of RVs but also their scattering around a random point $m_{X}, m_{Y}$. Thus, if an RV $X$ little deviates from its mathematical expectation, then the covariance will be small, despite the dependence between the RVs $X$ and $Y$. Therefore, the numerical characters of the dependence of RVs $X$ and $Y$ are the correlation coefficient, and not scattering. Therefore, if $\rho=0$, then the coordinates of the two-dimensional normal random vector are independent.

It is important to correctly evaluate the statistical significance of the correlation coefficient, that is, the degree of linear dependence between the sample characteristics. If its size $n$ is large, then the Student's statistics will be used for the correlation coefficient

$$
t=\frac{|\rho| \sqrt{n-2}}{\sqrt{\Delta_{\rho}}}, \quad \Delta_{\rho}=1-\rho^{2}
$$


with $n-2$ degrees of freedom. If at the sample size $n<30$ the value $\rho \geq 0.8$ is obtained, then Fisher's distribution statistics will be applied:

$$
z=\frac{1}{2} \ln \frac{1+\rho}{1-\rho}
$$

The transformation of $R V s$ and the Jacobian determinants. Arithmetic operations on the RVs by the transformation $Z=g(X, Y)$ are used to form two-dimensional systems [18]. At the same time, the theorem that the expectation of the sum of several RVs is equal to the sum of the mathematical expectations of these variables is important. This theorem is widely used because it does not impose any restrictions on the RVs included in the sum, that is, they can be dependent and independent. So as for independent $\mathrm{RVs}$, the equation $E_{(X \cdot Y)}=E_{X} \cdot E_{Y}$ is true only for independent $\mathrm{RVs}$, that the transformation of the following type is incorrect:

$$
E_{X^{2}}=E_{(X \cdot X)}=E_{X} \cdot E_{X}=\left(E_{X}\right)^{2}
$$

as both factors are mutually dependent and the multiplication theorem cannot be used in this case. If the transformation (29) was true, the difference $E_{X^{2}}-\left(E_{X}\right)^{2}$ would be equal to zero, that cannot be true, because, in a system of random nature, the variance is

$$
D_{X}=E_{X^{2}}-\left(E_{X}\right)^{2}>0
$$

In the general case, the theorem on the mathematical expectation of the product of RV has the following equation:

$$
E_{(X \cdot X)}=E_{X} \cdot E_{X}+\rho \cdot \sigma_{X} \cdot \sigma_{Y} .
$$

Therefore, if a vector $\vec{Z}=(X, Y)$ with the probability density function $f_{(X, Y)}(x, y)$ is given and it is necessary to find the distribution law of the transformed $\mathrm{RV} Z=g(X, Y)$, then it is enough to construct an appropriate single-valued view of the vector $\vec{Z}=(X, Y)$ into a vector $\vec{W}=(W, V)$ and find the probability density function of the vector $\vec{W}[21]$ :

$$
Z=g(X, Y)=\left\{\begin{array}{cll}
X+Y & \text { and } & X-Y \\
X \cdot Y & \text { and } & X / Y
\end{array} .\right.
$$

The formulas for calculating the probability density functions $f_{Z}(z)$ transformed according to algorithms (32) of correlated and uncorrelated RVs are shown in Figure 3. In general, the probability density functions are transformed according to the following rule. Let two direct transformations $Z=g(X, Y)$ (inverted $\left.X=g_{X}^{-1}(Z, V), Y=g_{Y}^{-1}(Z, V)\right)$ and $V=v(X, Y)$ take place at the RVs $X$ and $Y$ with joint two-dimensional probability density function $f_{(Z, V)}(z, v)$ and let us find the probability density function $f_{(Z, V)}(z, v)$ of two-dimensional $\mathrm{RVs}(Z, V)$. The following relationship is true in the differential equation:

$$
f_{(Z, V)}(z, v)=f_{(X, Y)}\left(g_{X}^{-1}(z, v), g_{Y}^{-1}(z, v)\right)\left|\frac{\partial(x, y)}{\partial(z, v)}\right|=f_{(X, Y)}\left(g_{X}^{-1}(z, v), g_{Y}^{-1}(z, v)\right)|J(z, v)|,
$$

where $J(z, v) \cdot J(x, y)=1$.

To find the distribution law of transformations (40) for independent RVs, we should switch from the system of RVs $(X, Y)$ to the system of $\operatorname{RVs}(Z, X)$ or $(Z, Y)$ and carry out integration according to the 
formula (12). To do this, we have to solve the equation $Z=g(X, Y)$ for one of the transitions from the system RVs $(X, Y)$ to the system of RVs $(Z, X)$ or $(Z, Y)$. For the case $y=w(z, x)$, it is given that

$$
f\left(x, g^{-1}(z, x)\right) d x=g(z, x) d z \Rightarrow g(z, x)=f\left(x, g^{-1}(z, x)\right)\left|\frac{d y}{d z}\right|=f\left(x, g^{-1}(z, x)\right)|J|,
$$

and for the case $x=q(z, y)$,

$$
g(z, y)=f\left(g^{-1}(z, y), y\right)\left|\frac{d x}{d z}\right| .
$$

Then the probability density function is calculated as an integral

$$
f_{Z}(z)=\int_{-\infty}^{\infty} g(z, x) d x=\int_{-\infty}^{\infty} g(z, y) d y .
$$

Mutual single-valued direct and inverse transformations and Jacobian determinants of

\begin{tabular}{|c|c|c|c|}
\hline Direct transformation & Inverse transformation & \multicolumn{2}{|l|}{ Jacobian determinants of transformations } \\
\hline$Z=g(X, Y)$ & $g_{X, Y}^{-1}(Z, V)$ & $(X, Y) \rightarrow\left(Z, V\left\{\begin{array}{l}X \\
Y\end{array}\right)_{:}|J(z, v)|=\mid \begin{array}{ll}\partial x / \partial z & \partial \\
\partial y / \partial z & \partial\end{array}\right.$ & $\begin{array}{l}\partial x / \partial v \\
\partial y / \partial v\end{array}$ \\
\hline $\begin{aligned} Z & =X+Y \\
V & =X\end{aligned}$ & $\begin{array}{l}X=V \\
Y=Z-V\end{array}$ & \multicolumn{2}{|l|}{ 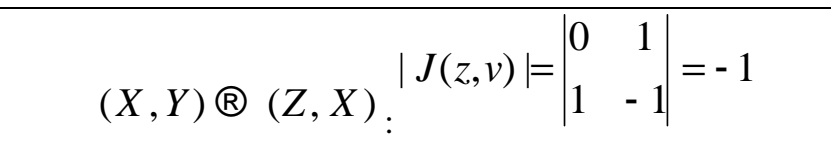 } \\
\hline $\begin{aligned} Z & =X+Y \\
V & =Y\end{aligned}$ & $\begin{array}{l}Y=V \\
X=Z-V\end{array}$ & \multicolumn{2}{|l|}{$(X, Y) \rightarrow(Z, Y):|J(z, v)|=\left|\begin{array}{cc}1 & 1 \\
0 & -1\end{array}\right|=-1$} \\
\hline $\begin{array}{l}Z=X-Y \\
X=X\end{array}$ & $\begin{array}{l}X=Y \\
Y=X-Z\end{array}$ & \multicolumn{2}{|l|}{$(X, Y) \rightarrow(Z, X)\left|{ }_{:}\right| J(z, v)|=| \begin{array}{cc}0 & 1 \\
-1 & 1\end{array} \mid=1$} \\
\hline $\begin{array}{l}Z=X-Y \\
Y=Y\end{array}$ & $\begin{array}{l}X=X \\
Y=X-Z\end{array}$ & \multicolumn{2}{|l|}{$(X, Y) \rightarrow(Z, Y):|J(z, v)|=\left|\begin{array}{cc}0 & 1 \\
-1 & 1\end{array}\right|=1$} \\
\hline $\begin{aligned} Z & =X \cdot Y \\
V & =X\end{aligned}$ & $\begin{array}{l}X=V \\
Y=Z / V\end{array}$ & \multicolumn{2}{|l|}{$(X, Y) \rightarrow(Z, X):|J(z, v)|=\left|\begin{array}{ll}y & x \\
0 & 1\end{array}\right|=\frac{1}{y}$} \\
\hline $\begin{aligned} Z & =X \cdot Y \\
V & =Y\end{aligned}$ & $\begin{array}{l}X=Z / V \\
Y=V\end{array}$ & \multicolumn{2}{|l|}{$(X, Y) \rightarrow(Z, Y):|J(z, v)|=\left|\begin{array}{ll}y & x \\
0 & 1\end{array}\right|=\frac{1}{y}$} \\
\hline $\begin{array}{l}Z=X / Y \\
V=X\end{array}$ & $\begin{array}{l}X=V \\
Y=V / Z\end{array}$ & \multicolumn{2}{|l|}{$(X, Y) \rightarrow(Z, X):|J(z, v)|=\left|\begin{array}{cc}0 & 1 \\
1 / x & -z / x^{2}\end{array}\right|=$} \\
\hline $\begin{aligned} Z & =X / Y \\
V & =Y\end{aligned}$ & $\begin{array}{l}X=Z \cdot V \\
Y=V\end{array}$ & \multicolumn{2}{|l|}{$(X, Y) \rightarrow(Z, Y):|J(z, v)|=\left|\begin{array}{cc}0 & 1 \\
1 / x & -z / x^{2}\end{array}\right|=y$} \\
\hline
\end{tabular}
transformations of the RVs for the sum, difference, product, and ratio of RVs are given in Table 1.

Table 1

Let us demonstrate the transformation algorithm by the example of the transition from the Cartesian coordinate system $x, y$ to the polar coordinate system $r, \varphi$ :

$$
(x, y) \rightarrow(r, \varphi), r>0, \varphi \in(-\pi, \pi),
$$


For which the Jacobian determinant of the transformation is put down as follows:

Thus,

$$
\frac{\partial(r, \varphi)}{\partial(x, y)}=\operatorname{det}\left(\begin{array}{ll}
\partial r / \partial x & \partial r / \partial y \\
\partial \varphi / \partial x & \partial \varphi / \partial y
\end{array}\right)=\operatorname{det}\left(\begin{array}{cc}
x / r & y / r \\
\frac{-y / x^{2}}{1+(y / x)^{2}} & \frac{1 / x}{1+(y / x)^{2}}
\end{array}\right)=\frac{1}{r} \Rightarrow|J|=r
$$

$$
f_{(R, \varphi)}(r, \varphi)=r f_{(X, Y)}(x=r \cos \varphi, y=r \sin \varphi)
$$

If the Cartesian coordinates are distributed under the normal law $N(0,1)$, then in spherical coordinates we have

$$
f_{(R, \varphi)}(r, \varphi)=\frac{r}{2 \pi} e^{-\frac{x^{2}+y^{2}}{2}}=\frac{r}{2 \pi} e^{-\frac{r^{2}}{2}}=f_{R}(r) \cdot f_{\varphi}(\varphi)
$$

The uniformly distributed two-dimensional system of $R V s$. The probability density function $f_{(X, Y)}(x, y)$ of the joint distribution of two RVs is equal to:

$$
f_{(X, Y)}(x, y)=\frac{1}{S}, \quad\left\{\begin{array}{l}
0 \leq x \leq x_{\max } \\
0 \leq y \leq y_{\max }
\end{array} .\right.
$$

The surface of the function (41) has the shape of a rectangular parallelepiped, one of the vertices of which is aligned with the origin of coordinates in the plane $X o Y$ with $x_{\max }, y_{\max }$ long sides, so the region of the rectangle is $S=x_{\max } \cdot y_{\max }$. The marginal probability density function is equal to:

$$
f_{X}(x)=\int_{0}^{x_{\max }} f_{(X, Y)}(x, y) d y=\frac{1}{S} \int_{0}^{y_{\max }} d y=\frac{1}{x_{\max }}, \quad f_{Y}(y)=\int_{0}^{x_{\max }} f_{(X, Y)}(x, y) d x=\frac{1}{y_{\max }} .
$$

If the two-dimensional $\mathrm{RV}(X, Y)$ is uniformly distributed in a circle with a radius $R$, then the joint probability density function is equal to:

$$
f(x, y)= \begin{cases}\frac{1}{\pi R^{2}}, & \text { if } \quad x^{2}+y^{2} \leq R^{2}, \\ 0, & \text { if } \quad x^{2}+y^{2}>R^{2} .\end{cases}
$$

The marginal probability density functions of both components in this case at $|x| \leq \sqrt{R^{2}-y^{2}}$ and $|y| \leq \sqrt{R^{2}-x^{2}}$ are equal to:

$$
\begin{aligned}
& f_{Y}(y)=\int_{-\infty}^{\infty} f(x, y) d x=\frac{1}{\pi R^{2}} \int_{-\sqrt{R^{2}-y^{2}}}^{\sqrt{R^{2}-y^{2}}} d x=\left.\frac{1}{\pi R^{2}} x\right|_{-\sqrt{R^{2}-y^{2}}} ^{\sqrt{R^{2}-y^{2}}}=\frac{2}{\pi R} \sqrt{1-\left(\frac{y}{R}\right)^{2}}, \\
& f_{X}(x)=\int_{-\infty}^{\infty} f(x, y) d y=\frac{1}{\pi R^{2}} \cdot \int_{-\sqrt{R^{2}-x^{2}}}^{\sqrt{R^{2}-x^{2}}} d x=\left.\frac{2}{\pi R^{2}} \cdot y\right|_{-\sqrt{R^{2}-x^{2}}} ^{\sqrt{R^{2}-x^{2}}}=\frac{2}{\pi R} \sqrt{1-\left(\frac{x}{R}\right)^{2}} .
\end{aligned}
$$

Let us verify the condition of independence of RVs:

$$
\frac{1}{\pi R^{2}} \neq \frac{1}{2 R} \sqrt{1-\left(\frac{x}{R}\right)^{2}} \cdot \frac{1}{2 R} \sqrt{1-\left(\frac{y}{R}\right)^{2}} \Rightarrow f(x, y) \neq f_{X}(x) \cdot f_{Y}(y) .
$$

Thus, although the joint distribution function (51) is divided into the product of two functions, one of which depends only on one variable, and the other one is dependent on the other RV (formula (44)), the condition of equality of the function $f_{(X, Y)}(x, y)$ of the product $f_{X}(x) \cdot f_{Y}(y)$ is not met, that is, the RVs 
with joint distribution (43) are dependent. Let us calculate the probability density functions of conditional probability distributions:

$$
\begin{aligned}
& f_{X}(x \mid y)=\frac{f(x, y)}{f_{Y}(y)}=\frac{\frac{1}{\pi R^{2}}}{\frac{2}{\pi R} \sqrt{1-(y / R)^{2}}}=\frac{1}{2 R}\left(1-(y / R)^{2}\right)^{-1 / 2} \neq f_{X}(x) \\
& f_{Y}(y \mid x)=\frac{f(x, y)}{f_{X}(x)}=\frac{\frac{1}{\pi R^{2}}}{\frac{2}{\pi R} \sqrt{1-(x / R)^{2}}}=\frac{1}{2 R}\left(1-(x / R)^{2}\right)^{-1 / 2} \neq f_{Y}(y)
\end{aligned}
$$

Here, the fact that $f(x, y)=0$ at $x^{2}+y^{2}>R^{2}$, therefore $f_{X}(x \mid y)=0$ at $|x|>\sqrt{R^{2}-y^{2}}$ and similarly: $f_{Y}(y \mid x)=0$ at $|x|>\sqrt{R^{2}-y^{2}}$, is not taken into account. Note that the geometric probability calculation can be used for uniform distribution.

If $X=0$, then the RV $Y$ can take an arbitrary value from the range $[-R ;+R]$ with the same probability; if $X= \pm R$, then the RV $Y$ can take only a single value $Y=0$. To make sure, whether there is a functional relationship between the RVs $X$ and $Y$ the considered problem, we should calculate the correlation coefficient (normalized covariance). In the case of uniform generation of random points in the area of the circle with the radius $R$, the correlation moment is equal to:

$$
\mathrm{K}_{C}=\iint_{\left(C=\pi r^{2}\right)} x y f_{C}(x, y) d x d y=\frac{1}{\pi r^{2}} \iint_{\left(C=\pi r^{2}\right)} x y d x d y
$$

If the origin of the Cartesian coordinate system is aligned with the center of the circle, the area of which is uniformly filled with random points, then, taking into account the symmetry between the mutually opposite quadrants (sectors), we conclude that the correlation moment is equal to zero, that is, in this system, the RVs $X$ and $Y$ are uncorrelated, yet dependent. The correlation coefficient does not characterize any dependence, but a linear dependence, and in the borderline case $Y=k X+$ const, it is equal to $\rho= \pm 1$.

Normally distributed two-dimensional system of $\boldsymbol{R} V \mathbf{s}^{\square)}$. Continuous multidimensional systems described by the normal distribution, based on the concept of a random vector, are widely used in physical modeling and many engineering applications of probability theory. It was substantiated that a continuous random vector $(X, Y)$ as a two-dimensional system of correlated RVs $X \sim N\left(m_{X}, \sigma_{X}\right)$ i $Y \sim N\left(m_{Y}, \sigma_{Y}\right)$ with unconditional mathematical expectations $m_{X, Y}$ and unconditional standard deviations $\sigma_{X, Y}$ is distributed according to normal law if the joint probability density function has the form of $[1 ; 14]$ :

$$
f_{(X, Y)}(x, y)=\frac{1}{2 \pi \sqrt{\Delta_{\rho}}} \exp \left\{-\frac{1}{2}\left[\frac{\left(x-m_{X}\right)^{2}}{\sigma_{X}{ }^{2}}+\frac{\left(y-m_{Y}\right)^{2}}{\sigma_{Y}{ }^{2}}-2 \rho \frac{\left(x-m_{X}\right)}{\sigma_{X}} \frac{\left(y-m_{Y}\right)}{\sigma_{Y}}\right]\right\},
$$

or in a more convenient vector and matrix notation

$$
f_{(X, Y)}(x, y)=\frac{1}{2 \pi \sqrt{\Delta_{\rho}}} \exp \left(-\frac{1}{2 \Delta_{\rho}}\left[\left(\begin{array}{c}
x-m_{X} \\
y-m_{Y}
\end{array}\right)^{T}\left(\begin{array}{cc}
\sigma_{X}{ }^{-2} & \frac{\rho}{\sigma_{X} \sigma_{Y}} \\
\frac{\rho}{\sigma_{X} \sigma_{Y}} & \sigma_{Y}{ }^{-2}
\end{array}\right)\left(\begin{array}{c}
x-m_{X} \\
y-m_{Y}
\end{array}\right)\right]\right)
$$

More detailed information about the statistical and probabilistic normal distribution of binary systems is available in the monograph [8] 
It follows from (56) that the RVs $X$ and $Y$ are independent, only when the correlation coefficient is equal to $\rho=0$. Then

$$
f_{(X, Y)}(x, y)=\frac{1}{2 \pi \sigma_{X}^{*} \sigma_{Y}^{*}} \exp \left(-\frac{1}{2}\left[\frac{x^{2}}{\sigma_{X}^{*^{2}}}+\frac{y^{2}}{\sigma_{Y}^{* 2}}\right]\right)=f_{X}(x) \cdot f_{Y}(y), \quad \sigma_{X, Y}^{*}{ }^{2}=\sigma_{X, Y}^{2} \sqrt{1-\rho^{2}} .
$$

The covariance of the RVs in a two-dimensional system with normally distributed RVs is calculated as a double integral:

$$
\operatorname{cov}(x, y)=\int_{-\infty-\infty}^{\infty} \int_{-\infty}^{\left(x-m_{X}\right)\left(y-m_{Y}\right)} \frac{1}{2 \pi \sigma_{X} \sigma_{Y} \sqrt{\Delta_{\rho}}} \frac{1}{2 \pi \sigma_{X}^{*} \sigma_{Y}^{*}} \exp \left(-\frac{1}{2 \Delta_{\rho}}\left[\frac{x^{2}}{\sigma_{X}{ }^{2}}+\frac{y^{2}}{\sigma_{Y}^{2}}-\frac{2 \rho\left(x-m_{X}\right)\left(y-m_{Y}\right)}{\sigma_{X} \sigma_{Y}}\right]\right)
$$

In the general case of normal correlation, the RVs $X$ and $Y-\rho \frac{\sigma}{\sigma_{Y}} X$ or $X-\rho \frac{\sigma}{\sigma_{Y}} Y$ and $Y$ [14, page 350] are independent.

The integral probability density function of two standard normal values is equal to:

$$
F_{(X, Y)}(x, y)=\int_{-\infty}^{\frac{x-m_{X}}{\sigma_{X}}} \int_{-\infty}^{\frac{y-m_{Y}}{\sigma_{Y}}} \frac{1}{2 \pi \sqrt{\Delta_{\rho}}} \exp \left(-\frac{x^{2}-2 \rho x y+y^{2}}{2 \Delta_{\rho}}\right) d x d y
$$

If the full square is highlighted in the exponent

$$
\frac{1}{2 \Delta_{\rho}}\left(x^{2}-2 \rho x y+y^{2}\right)=\frac{1}{2 \Delta_{\rho}}\left(x^{2} \Delta_{\rho}+(y-\rho x)^{2}\right),
$$

the subintegral function in (52) can be represented as a product of

$$
\begin{aligned}
& f_{(X, Y)}(x, y)=\frac{1}{2 \pi \sqrt{\Delta_{\rho}}} \exp \left(-\frac{x^{2}-2 \rho x y+y^{2}}{2 \Delta_{\rho}}\right)= \\
& =\frac{1}{\sqrt{2 \pi \Delta_{\rho}}} e^{-\frac{(y-\rho x)^{2}}{2 \sqrt{\Delta_{\rho}}}} \cdot \frac{1}{\sqrt{2 \pi}} e^{-\frac{x^{2}}{2}}=f_{Y}(y \mid X=x) \cdot f_{X}(x)
\end{aligned}
$$

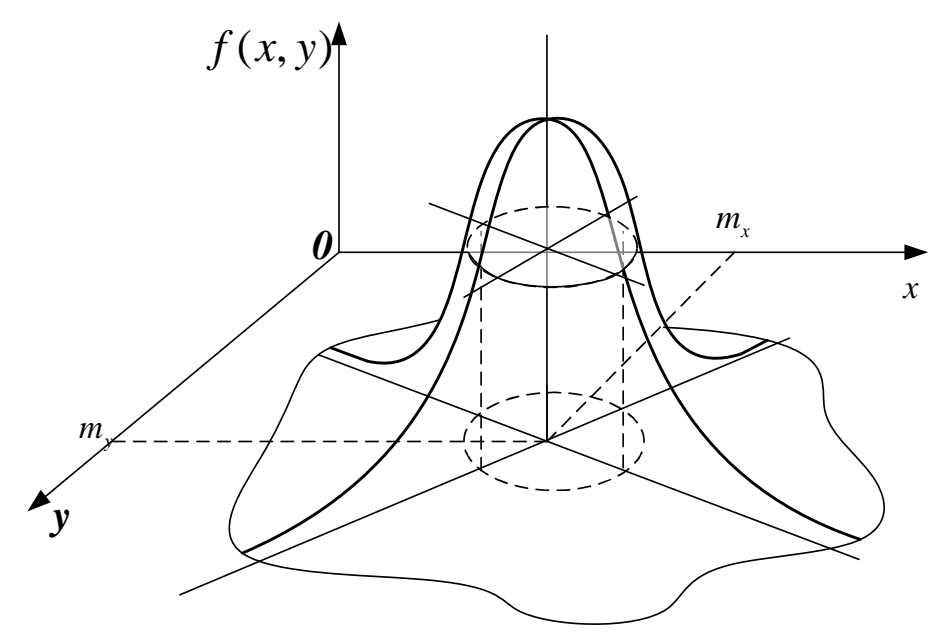

Fig. 3. Illustration of the graph of the function $f_{(X, Y)}(x, y)$

The graph of the function $f_{(X, Y)}(x, y)$ is shown in Fig. 3. The surface vertex is projected onto a plane $x 0 y$ as a point $\left(m_{X}, m_{Y}\right)$. The cross-section of the surface plane parallel to the plane $x 0 y$ is the 
ellipse of equal probability $f_{(X, Y)}(x, y)=$ const, the center of which is a point $\left(m_{X}, m_{Y}\right)$. The ellipse of equal probability is also called the scattering ellipse that is why the point $\left(m_{X}, m_{Y}\right)$ is also called the scattering center. If the components $X$ and $Y$ of the two-dimensional RVs are independent variables, then the ellipse axes are parallel to the coordinate axes. For uncorrelated RVs, the ellipse has a shape of a circle.

As the joint probability density function is

$$
\left.f_{(X, Y)}(x, y)=f_{Y \mid X}(y \mid x) f_{X}(x)=f_{X \mid Y}(x \mid y) f_{Y}(y)\right),
$$

then the conditional probability density functions will be equal to:

$$
\begin{aligned}
& f_{Y \mid X}(y \mid x)=\frac{1}{\sigma_{X} \sqrt{2 \pi \Delta_{\rho}}} \exp \left\{-\frac{1}{2 \Delta_{\rho}}\left[\rho \frac{\left(x-m_{X}\right)}{\sigma_{X}{ }^{2}}+\frac{\left(y-m_{Y}\right)}{\sigma_{Y}}\right]^{2}\right\}, \\
& f_{X \mid Y}(x \mid y)=\frac{1}{\sigma_{Y} \sqrt{2 \pi \Delta_{\rho}}} \exp \left\{-\frac{1}{2 \Delta_{\rho}}\left[\frac{\left(x-m_{X}\right.}{\sigma_{X}}+\rho \frac{\left(y-m_{Y}\right)}{\sigma_{Y}}\right]^{2}\right\},
\end{aligned}
$$

wherefrom the one-dimensional law of normal distribution follows

$$
f_{Y \mid X}(y \mid x)=\frac{1}{\sigma_{X} \sqrt{2 \pi \Delta_{\rho}}} \exp \left\{-\frac{\left[y-m_{y \mid x}\right]^{2}}{2 \sigma_{Y \mid X}^{2}}\right\}
$$

with conditional mathematical expectations and variance

$$
\begin{array}{lll}
m_{Y \mid X}=m_{Y}+r \frac{\sigma_{Y}}{\sigma_{X}}\left(x-m_{X}\right) & \text { and } & \sigma_{Y \mid X}{ }^{2}=\sigma_{Y}{ }^{2} \Delta_{\rho} . \\
m_{X \mid Y}=m_{X}+r \frac{\sigma_{X}}{\sigma_{Y}}\left(y-m_{Y}\right) & \sigma_{X \mid Y}{ }^{2}=\sigma_{X}{ }^{2} \Delta_{\rho} .
\end{array}
$$

If $\Delta_{\rho}<1$ then the variance of conditional mathematical expectations is less than unconditional ones; at the same time, it decreases to the extent, to which the correlation between $\mathrm{RVs} X$ and $Y$ increases. Thus, RVs $X$ and $Y$ will have the following conditional distributions:

$$
\begin{aligned}
& X \mid Y_{\sim} N\left(m_{X}+r \frac{\sigma_{X}}{\sigma_{Y}}\left(y-m_{Y}\right), \sigma_{X}{ }^{2} \Delta_{\rho}\right), \\
& Y \mid X_{\sim}^{N} N\left(m_{X}+r \frac{\sigma_{X}}{\sigma_{Y}}\left(y-m_{Y}\right), \sigma_{Y}{ }^{2} \Delta_{\rho}\right) .
\end{aligned}
$$

The graphs of conditional mathematical expectations are straight lines (straight lines of mean quadratic regressions) that intersect at the point $\left(m_{X}, m_{Y}\right)$ in the center of the joint distribution of RVs $X$ and $Y$.

Thus, as follows from (59) and (60), the mathematical expectation linearly depends on the condition, whereas the variance does not depend on the condition. Therefore, the value $m_{X \mid Y}$ is called the conditional mathematical expectation of $\mathrm{RV} X$, provided that either $Y=y$ or the regression of $\mathrm{RV} X$ on the variable $Y$ with a regression coefficient $\rho \frac{\sigma_{X}}{\sigma_{Y}}$. The variable $\sigma_{X, Y}{ }^{2} \Delta_{\rho}$ is also called the residual variance of the $\mathrm{RV}$ of one RV against another. Residual variance characterizes the value of an error arising from the replacement, for example from the replacement of RV $X$ with a linear function $g(Y)=a Y+b$.

According to Cauchy-Schwarz inequality, $-1<\rho<+1$. For values $\rho= \pm 1$, there are linear dependencies with positive and negative slopes, for which there is no residual variance and the 
corresponding error is equal to zero, that is, the RV $X$ is accurately represented by a linear function $g(Y)=a Y+b$ of the RV $Y$. These cases correspond to the transformation of the quadratic function $Q(x, y)$ into linear functions. For linear relationships between the random variables that form a twodimensional system, the function $g(Y)$ will be the best approximation of the RV $X$ in terms of the leastsquares method, if the mathematical expectation is

$$
M[X-g(Y)]^{2} \rightarrow \min .
$$

\section{Conclusion}

A methodological analysis of primary sources for the formulation of basic laws of the twodimensional distribution of random variables, which are most relevant for the correct interpretation of the data of physical experiments. This article is introductory and provides for its continuation, where it is planned to reflect the following important sections,as: A two-dimensional system of Cauchy-distributed RVs; A two-dimensional system of Rayleigh-distributed RVs; A three-dimensional system of Maxwelldistributed RVs; Statistical modeling of the composition of the RVs; The composition of Gaussian RVs; The composition of two normally distributed random vectors; The composition of Rayleigh-distributed $\operatorname{RVs} X_{i} \sim \operatorname{Ra}\left(\sigma_{i_{i}}, i=1,2\right)$ with the scattering region of values $\left(0<x_{i}<\infty\right)$; The distribution of the sum of Maxwell-distributed RVs; The Distribution Regularities of the Difference Module $R=|X-Y|$; Statistical Regularities of the Composition of Normally Distributed RVs of the Type $X+Y^{2}$; Distribution of the Sum $Z=\left.(X+Y)\right|_{\rho \neq 0}$ of Correlated Normally Distributed RVs; About some features of the distribution of a two-dimensional system of dependent RVs; Statistical Modeling of the Correlation $Z=\frac{X}{Y}$ of RVs in a Two-dimensional System and Modeling of the Distribution Parameters of the Product $Z=X \cdot Y$ of RVs in a Two-Dimensional System.

1. Jonson N., Kotz S., Balakrishnan N. Continuous Univariate Distributions.1995, Wiley, New York.

2. Kolmogorov A.N., Foundations of the Theory of Probability, Chelsea Pub Co., 2nd Edition 1960.

3. Frolov A. N. Limit theorems of probability theory. St. Petersburg, 2014, 152 pages

4. Shevchuk V. Calculation of dynamic errors of intelligent measuring systems. Moscow: Fizmatlit, 2008

5. Nadarajah, S., and Kibria, B. M. G. (2006). On the ratio of generalized Pareto random variables. Stochastic Environmental Research \& Risk Assessment, 206-212.

6. Purcell E. Electricity and Magnetism.v.2 McGraw-Hill Book Company.

7. Tucker H. An Introduction to Probability and Mathematical Statistics.NY Academic Press 1962.

8. Tong Y.L. The Multivariate Normal Distribution. Springer Series in Statistics. Springer-Verlag.

9. Hegge F. A Simple Zener Diode Voltage Regulator.Journal of the Experimental Analysis of Behavior Volume 8, Issue 1,1965.

10. Cramer H. RVs and Probability Distributions. Cambridge Tracts in Mathematics and Mathematical Physics, No36, Cambridge University Press, 1962.

11. Cooper G., McGillem C. Probabilistic Methods of Signal and System Analysis, Oxford University Press.

12. Deserno M. The probability density of the sum of two uncorrelated RVs is not necessarily the convolution of its two marginal densities; $\underline{\text { https://www.cmu.edu/biolphys/deserno/pdf/uncorrelated-sum-pdensity.pdf. }}$.

13. Mari D., Kotz S. Correlation and Dependence. Empirical College Press.2001.

14. Bernstein S. N. Probability theory. Moscow-Leningrad: 1927.

15. Pearson K. Contributions to the Mathematical Theory of Evolution.II.Skew Variations in Homogeneous Material. Philosophical Transactions Of the Royal Society of London.Ser.A.-1895.-Vol.186.-P.343-414. http://rsta.royalsocietypublishing.org/content/roypta/186/343.full.pdf; The problem of the random walk.Nature.1905.-vol.72.Issue 1866,-p.342.

16. Lehmann E.L. Some concepts of dependence, Ann. Math.Statist. 37,1137-1153 (1966). 
17. Louis de Broglie. Les incertitudes Heisenberg et l'interprétation probabiliste de la mécanique undulatory, Gauthier-Villars, 1982.

18. Jaroszewicz S., Korzen M. Arithmetic operations on independent RVs: a numerical approach. SIAM J.Sci Comput. Vol.34, No3, A1241-1265,2012.

19. Springer, M. The Algebra of RVs, Wiley, New York, 1984.

20.Springer M., Thompson W. The distribution of products of beta, gamma, and Gaussian RVs. SIAM Journal on Applied Mathematics. 18 (4): 721-737, (1970); The distribution of products of independent RVs. SIAM Journal on Applied Mathematics. 14 (3): 511-526,1966.

21. Seijas-Magic A. An approach to Distribution of the Product of Two Normal Variables. Discussions Mathematics. Probability and Statistics vol.32,87-99 (2012).

П. Кособуцький

Національний університет “Львівська політехніка"

\section{МЕТОДИЧНІ АСПЕКТИ СТАТИСТИЧНОГО МОДЕЛЮВАННЯ ДВОВИМІРНИХ СИСТЕМ ІЗ ВИПАДКОВИМИ ДАНИМИ}

(ㄱ Кособуиький П., 2020

Як свідчить аналіз літературних джерел, статистичній обробці результатів вимірювань не завжди приділясться належна увага. На превеликий жаль, відповідні алгоритми часто обмежуються спрощеними статистичними процедурами, без належного обгрунтування цільової функції, в тому числі для перевірки якості опрацювання випадкових даних. Тому автор планус опублікувати серію статей із статистичного моделювання, які включатимуть результати оригінальних досліджень самого автора та інших. В цій статті, розглянуті методичні аспекти статистичного моделювання двовимірних систем із випадковими даними, дане фізичне обгрунтування кореляційних закономірностей статистичних співвідношень між випадковими величинами, оскільки задача встановлення закону розподілу випадкової величини має практичний інтерес 3 точки зору моделювання статистичних закономірностей моделі «сигнал+шум».

Ключові слова: Двовимірні системи, умовні ймовірності, Залежні та незалежні, Якобійські детермінанти, кореляція та трансформація буксирної мірності 